\title{
Saturation Throughput Analysis for an Ideal Loss Differentiated DCF over Wireless Lan
}

\author{
Hongyuan Chen, \\ School of Electronic and Optical Engineering \\ Nanjing University of Science and Technology (NJUST) \\ Nanjing, China, 210094 \\ chy@mail.njust.edu.cn
}

\author{
Runtong Geng \\ School of Electronic and Optical Engineering \\ Nanjing University of Science and Technology (NJUST) \\ Nanjing, China, 210094 \\ grtzhuxian@163.com
}

\begin{abstract}
In a real wireless channel, both a channel bit error and a collision between competition stations can cause a data frame loss. Moreover, DCF always assumes that a data frame loss is due to the collision and the contention window will double in next transmission. In fact, we think that the contention window shall be difference between channel bit error and collision. In this paper, we propose a ideal mechanism LD-DCF which can completely differentiate between the two reasons of a data frame loss. An analytical model is given to demonstrate the efficiency of LD-DCF. NS-2 simulation is also used to validate the LD-DCF. By the theoretical and simulation results, the LD-DCF can improve the throughput significantly, especial for the high channel bit error and low contending stations (i.e. the low network traffic).
\end{abstract}

Keywords- IEEE 802.11, CSMA/CA, DCF, Collision Aware

\section{INTRODUCTION}

The Distributed Contention Function (DCF) is the main medium access scheme in the MAC layer of IEEE 802.11 [1] Specifically, the DCF employs a Carrier Sense Multiple Access with Collision Avoidance (CSMA/CA) with a binary exponential backoff procedure. So many contributions have taken attention on the performance analysis of the DCF and they only focused on how to optimize the contention window. In the following, we discuss another issue, how to differentiate between the collision and channel bit error, and why the issue is important.

In the DCF, after a data frame is transmitted, the fact that a corresponding ACK frame isn't received in a predefined timeout interval shall cause data frame loss recognition. The DCF assumes that all data frame losses are caused by the data collision when transmitting. As a result, the next contention window shall be doubled based on the current contention window after a data loss. The contention window doubling can not only partly reduces the collision probability but also enlarge the waiting time for accessing the channel (so as to waste the channel bandwidth). In fact, in real wireless environment, the data frame loss can be caused by the imperfect wireless channel. That is, there are two reasons, data collision and channel bit error, which would result in the data frame loss when transmitting.

Intuitionally, it should respond the different actions for the data collision and the channel bit error because these two reasons have the essential differences. So many competition stations transmit the data frame at the same time so that the data collision occurs. Consequently, it should reduces the transmission probability at the same time in the next transmission. Thus, the contention window increasing is the correct action for the data collision.

Let's take a look at the data frame loss due to the channel bit error. If the channel bit error occurs when a data frame is transmitting, at least at the current time, it shows that there is not so much congest because the station has obtained the right of the channel access. Since the network traffic may be relatively low, the DCF should retransmit the data frame as soon as possible but not the current action, double the contention window.

Based on the above discussion, we have to ask one question ourselves, is it necessary for the DCF to differentiate between the two reasons of a data frame loss? This article aims to clarify the question from the theoretical viewpoint. This is our motivation to tackle the question. We develop a mathematical model to demonstrate the necessity. Moreover, we also show that how much space can be improved by the differentiation. NS-2 simulation results further validate the model accuracy and the efficiency of the differentiated actions.

\section{IDEAL LOSS DIFFERENTIATION DCF}

The ideal loss differentiation DCF (for simplicity, we called it LD-DCF) is based on the original DCF described in [1]. Note that, the ideal differentiation can be completely aware of the data frame loss due to either the data collision or the channel bit error. As to how to differentiate the collision from the channel bit error, it is out of scope of this article.

The collision avoidance procedure of the DCF is a binary exponential backoff algorithm. The backoff counter is uniformly chosen from the range determined by the contention window at each transmission attempt. At the first transmission attempt, the contention window shall be CWmin. After each failed transmission, the contention window is doubled based on the current window. After a maximum contention window CWmax is reached, then it remains at $\mathrm{CWmax}$ for the successive failed retransmissions. In this article, we assume the MPDU can be always retransmitted until it is transmitted successfully.

The LD-DCF is slightly different from the original DCF described as the above discussion. The difference is the 
reaction to the dataframe loss. When data collision occurs, the LD-DCF doubles the contention window based on the current value of the window in order to avoid the collision once again during the next transmitting time. When the channel bit error causes the data frame loss, the LD-DCF should use the CWmin as the contention window in order to transmit the data faster.

The design of the different reactions is based on the following consideration. Since the data frame is loss due to only the channel bit error, the transmitter has obtained the right of the channel access through the backoff procedure. Once the access right is obtained, it is reasonable to resume the backoff procedure for the next transmission regardless of the MPDU (either a new MPDU transmission or the same MPDU retransmission).

\section{ANALYTICAL MODEL}

Our model assumes that there are $\mathrm{n}$ contending stations (STA) in the network and that the data traffic of each STA is under saturation. Each MPDU has the same payload size Ldata which includes the physical header. Since the channel bit error should be considered, we have to assume the wireless channel is not ideal. In this article, a Gaussian channel is assumed where each bit of one MPDU has the same bit error probability (BER) and bit errors are identically and independently distributed over the whole MPDU.

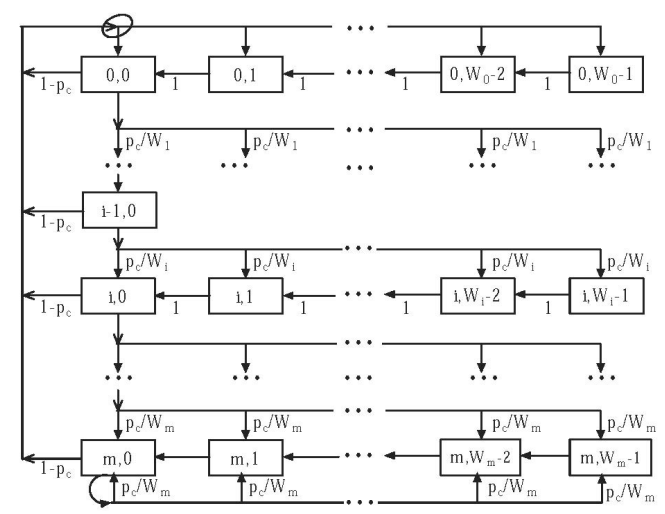

Figure 1. State transition diagram for LD-DCF

Our model is based on the Bianchi's model [2]. The Bianchi's model assumed that the channel is ideal. Here, we extend the Bianchi's model to consider the channel bit error. Even though the Ni's model [3] also took into account the channel bit error, our model is different from it due to the difference between the LD-DCF and the original DCF. As sated in the Bianchi's model [2], a bi-dimensional Markov chain $(\mathrm{s}(\mathrm{t}) ; \mathrm{b}(\mathrm{t}))$ is designed for the given tagged STA. $\mathrm{b}(\mathrm{t})$ represents the value of backoff counter while $s(t)$ is the backoff stage at model slot time t. The Markov chain is shown in Fig.1. Seemingly, it is same as the Bianchi's model. However, the reasons for each one step of state transition are different between our model and the Bianchi's model. Moreover, the throughput is computed based on the different ways because the channel bit error has to be taken into account.
Based on the description of LD-DCF, it can completely differentiate between the channel bit error and the data collision. Moreover, only the data collision can cause the contention window increasing. Thus, the channel bit error resets the contention window regardless of the retransmission.

In the i-th collision, the contention window is increased by double only when the data collision. That is, the backoff stage $\mathrm{i}-1$ goes into the stage $\mathrm{i}$ with collision probability pc. Obviously, pc is determined by the transmission probability $\tau$. Let $\tau$ be the probability that a STA transmits in a randomly chosen slot time, then $\mathrm{pc}=1-(1-\tau) \mathrm{n}-1$. In the Markov chain, the only non-null one-step transition probabilities are:

$$
\left\{\begin{array}{l}
P\{j, k \mid j, k+1\}=1, \quad k \in\left[0, W_{j}-2\right], j \in[0, m] \\
P\{j, k \mid j-1,0\}=p_{c} / W_{j}, \quad k \in\left[0, W_{j}-1\right], j \in[1, m] \\
P\{0, k \mid j, 0\}=\left(1-p_{c}\right) / W, k \in[0, W-1], j \in[0, m] \\
P\{m, k \mid m, 0\}=p_{c} / W_{\max }, \quad k \in\left[0, W_{\max }-1\right]
\end{array}\right.
$$
(We $\mathrm{m}=\log 2(\mathrm{Wmax} / \mathrm{W})$. Let $\mathrm{CWi}$ be the contention window in the $\mathrm{i}$-th retransmission attempt of the $\mathrm{CA}$ frame and $\mathrm{Wi}=\mathrm{CWi}+1$. Based on the backoff procedure, it obtains $\mathrm{Wi}=$ $2 \mathrm{iW}$; for $0 \leq \mathrm{i} \leq \mathrm{m}-1$ and $\mathrm{Wi}=2 \mathrm{~mW}$; for $\mathrm{m} \leq \mathrm{i} \leq \infty$.

Owing to the chain regularities, by imposing the normalization condition, we can solve the Markov chain and obtain the $t$. The detail computation process can be referred in [2].

$$
\tau=\frac{2}{1+W+p_{c} W \sum_{j=0}^{m-1}\left(2 p_{c}\right)^{j}}
$$

\section{SATURATION ThroughPUT}

Let $\delta$, TMPDU and TACK denote duration of an empty slot time, the time to transmit one MAC data packet (including MAC header, PHY header, and/or tail) and the time to transmit an ACK frame (including PHY header), respectively.

In order to compute the throughput, we have to analyze what happens in a model slot time. Four different events shall be noted in one model slot: 1) All STAs are during backoff decrement; 2) There is a successful transmission; 3) a data collision; 4) A failed transmission which excludes the failed transmission due to collision. Note that, in a model slot, after a data collision happens, maybe there also are some channel bit errors when transmitting the MPDU. For the case, we can always deem that the model slot is a data collision because the collision occurs prior to the channel bit error.

Based on the protocol, it is readily obtain the duration of one model slot for each event: 1) the duration is the empty slot time $\delta$; 2) a successful transmission time TS includes one MPDU transmission time and An ACK time plus corresponding IFSs; 3) if a collision occurs, the receiver doesn't response any ACK frames. Thus, a data collision time Tc includes the one MPDU transmission time plus an EIFS interval; 4) the channel error can cause the error of either one MPDU or an ACK the duration. For simplify, we always that a failed transmission time $\mathrm{Tf}$ includes one 
MPDU transmission time plus the corresponding ACK timeout and DIFS interval. Obviously, we have

$$
\left\{\begin{array}{l}
T_{s}=T_{M P D U}+S I F S+T_{A C K}+D I F S \\
T_{c}=T_{M P D U}+E I F S \\
T_{f}=T_{M P D U}+A C K_{\text {timeout }}+D I F S
\end{array}\right.
$$

If we can get the probability of each event, it can immediately obtain the throughput. First, we define that pe is the probability that a MPDU (with packet size Ldata) and/or an Imm-ACK frame (with packet size Lack) suffers errors. pe $=1-(1-B E R)$ Ldata(1-BER)Lack.

Let $\mathrm{Pb}$ denote the probability that there is at least one transmission in a model slot time. Thus, the probability of the first event is $1-\mathrm{Pb}$ because all STAs are waiting for transmission. Ps denotes the probability of a successful transmission in a model slot time. A successful transmission means that there is only one STA which transmits the MPDU and further the MPDU doesn't experience any collisions or channel bit errors. Correspondingly, a failed transmission means that the MPDU is transmitted incorrectly due to the channel bit error while only one STA transmits MPDU (if more than one STA transmit the MPDU, there is a collision). Let $\mathrm{Pf}$ denote the probability that there is a failed transmission and Pc denote the probability that there is a collision in a model slot, respectively. Obviously, Pc is determined by considering that there are more than one STAs transmitting MPDUs at the same model slot. Finally, we have

$$
\left\{\begin{array}{l}
P_{b}=1-(1-\tau)^{n} \\
P_{s}=n \tau(1-\tau)^{n-1}\left(1-p_{e}\right) \\
P_{f}=n \tau(1-\tau)^{n-1} p_{e} \\
P_{c}=1-(1-\tau)^{n}-n \tau(1-\tau)^{n-1}
\end{array}\right.
$$

In a model slot, only a successful transmission shall be the part of effective transmission bits. Thus, the throughput can be expressed as

$$
S=\frac{P_{s} \cdot P}{\left(1-P_{b}\right) \delta+P_{s} T_{s}+P_{c} T_{c}+P_{f} T_{f}}
$$

where $\mathrm{P}$ denotes the payload size of one MPDU. Note that Ldata consists of payload size P plus the MAC header (and MAC tail).

\section{PERformance EVAluation}

In this section, we implement the LD-DCF in the simulation tool NS-2 in order to validate our model. Except for the special statement, all the PHY and MAC parameters are compliant with the 802.11a standard. Two scenarios, moderate channel error $(\mathrm{BER}=10-5)$ and very noisy channel $(B E R=10-4)$, are investigated. The payload size of one MPDU is 2000 Bytes. The simulation results and numerical results are illustrated in the Fig.2. It is shown that our model can predict the LD-DCF performance accurately.

The LD-DCF can perform better than DCF under a small size network and the serious channel bit error. When the number of stations is lower than 5 and the channel bit error is
10-4, the LD-DCF significantly improve the throughput. In specially, the throughput of LD-DCF is multiple of DCF when there is only one stations. However, if the channel bit error is not so serious (10-5), the LD-DCF has almost lower throughput than DCF. The main reason is that the performance is dominantly determined by collision. Anyway, it has been demonstrated that a simple idea of LD-DCF can improve the throughput even for some partial working scenarios. If we revise the backoff more robust for collision, it is believed that the loss-collision differentiation DCF can improve the performance under any scenarios. Also, this is our future work.

\section{ANALYTICAL MODEL FOR DCF}

The analytical model of the DCF can be referred as the Ni's model [3]. Here, we briefly describe the model. For the DCF, both a collision and a channel bit error can cause the contention window double. Thus, the Markov chain can be same as that in Fig. 1 except for the probability pc and pc can be replaced by the probability pf. pf stands for the collision and/or error probability that a transmitted packet experiences. It is easily obtain the transmission probability tdc f for the DCF. The throughput has the same expression as the equation (5).

$$
\left\{\begin{array}{l}
p_{f}=1-\left(1-p_{c}\right)\left(1-p_{e}\right) \\
\tau_{d c f}=\frac{2}{1+W+p_{f} W \sum_{j=0}^{m-1}\left(2 p_{f}\right)^{j}}
\end{array}\right.
$$

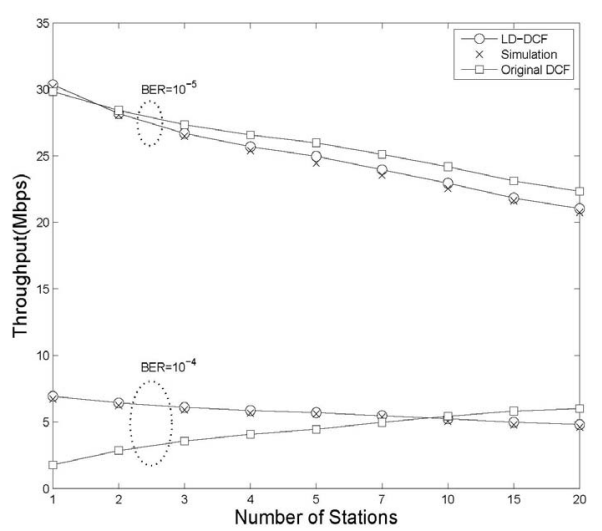

Figure 2. Aggregated Throughput: Rate $=54 \mathrm{Mbps}, \mathrm{CWmin}=7$, $\mathrm{CWmax}=1023$

\section{CONCLUSIONS}

This paper gives a simple idea to demonstrate the efficiency of loss-collision differentiation. Since a data loss is due to either the channel bit error or a data collision in a real wireless channel, it is reasonable that the DCF shall differentiate between two cases and have some different reactions on the cases. We simply propose LD-DCF which doubles the contention window only when a collision while resets the window into initial window for the channel bit error. An analytical model is given to compute the 
throughput of LD-DCF. Compared with the original DCF, the LD-DCF can greatly improve the throughput under the low network traffic and serious channel bit error.

\section{ACKNOWLEDGE}

This work was supported by ZiJing Scholar Plan of NJUST (No.AB41375).

\section{REFERENCES}

[1] IEEE, Standard for wireless lan medium access control (MAC) and physical layer(PHY) specification. IEEE802.11 WG, Aug, 1999.

[2] Bianchi, G., Performance analysis of the IEEE 802.11 distributed coordination function. IEEE J. Sel. Areas Commun., 18(3), Mar., pp. 535-547, 2000

[3] Ni, Q., Li, T., Turletti, T., and Xiao, Y., Saturation throughput analysis of error-prone 802.11 wireless networks. Wiley J. Wireless Commun. and Mobile Comput., 5(8), Dec., pp. 945-956, 2005 